\title{
Current Challenges in Early Intervention for Children with Autism Spectrum Disorder (ASD): A Narrative Review
}

\author{
Author \\ Costanza Colombi \\ ccolombi@umich.edu
}

\begin{abstract}
Autism Spectrum Disorder (ASD) is a neurodevelopmental disorder that involves global impairments in social skills and in verbal and non-verbal communication, as well as the presence of stereotyped patterns of behaviors and interests. ASD imposes an enormous burden on society including lifelong disability, high medical care, and increased mental health problems in caregivers. Evidence demonstrates that very early intervention delivered immediately after diagnosis during the first three years of life has higher impact on outcomes in comparison to later start. Early intervention has positive impact on verbal and nonverbal skills, adaptive skills, and quality of life of caregivers. Unfortunately, the majority of children in the world either start intervention after 4 years of age or never receive specialized intervention. The aim of this paper is to discuss current issues related to research and clinical implementation in the field of early intervention for young children with ASD. The paper will discuss intensity of services currently recommended, the need for effectiveness studies conducted outside the University settings, the inclusion/exclusion criteria used so far in intervention studies, and whether intervention should start during the first year of life.
\end{abstract}

Keywords: Autism Spectrum Disorder (ASD), Early Intervention, Challenges 


\section{Current Challenges in Early Intervention for Children with Autism Spectrum Disorder (ASD): A Narrative Review}

\section{Early Intervention for Children with Autism Spectrum Disorder (ASD): Current Challenges}

Autism is a neurodevelopmental disorder that involves global impairments in social skills and in verbal and non-verbal communication, as well as the presence of stereotyped patterns of behaviors and interests (DSM-5). Epidemiological reports indicate that the number of children diagnosed with autism spectrum disorders (ASD) is rising (Baxter et al., 2014). ASD affects approximately 1 in 68 children in the United States, according to the Center for Diseases Control (CDC). ASD imposes an enormous burden on society including lifelong disability, high medical care, and increased mental health problems in caregivers. Evidence demonstrates that very early intervention delivered immediately after diagnosis during the first three years of life has higher impact on outcomes in comparison to later start (Vivanti et al., 2015).

Early intervention has been correlated with improvement in verbal and nonverbal skills, adaptive skills, and quality of life of caregivers (L. K. Koegel, Koegel, Ashbaugh, \& Bradshaw, 2014). Since Lovaas's pioneering work started in the 60s (Lovaas, 1983), many intervention models have been developed and empirically evaluated. Approximately, evidence based early intervention models can be classified as based on the principle of behavioral analysis (Koegel et al., 2014), based on developmental and relationship models (Wetherby et al., 2014), or a fusion of behavioral strategies and relationshipfocused developmental models (Rogers et al., 2012). Despite the implementation of a specific model, the National Research Council (2001) recommends intervention intensity of at least $25 \mathrm{hrs}$. per week to be initiated as early as possible in life and immediately after diagnosis.
Many challenges are currently involved in early intervention research and in the consequent implementation of the various intervention models in the real world. Despite the National Research Council recommendations, several barriers prevent the implementation of early intervention in the first three years of life, including an average waiting list of three years from first concerns of parents and diagnosis (Christensen et al., 2012), scarcity of intervention providers, and lack of insurance coverage or State funding in some geographical area. Regarding the research arena, many questions needed to better guide best practice in the clinical work are still unanswered. These include, for example, optimal intensity of services, adaption of the interventions from the University setting to the real world, and explanations for the heterogeneity of outcomes in children with ASD. The aim of this paper is to discuss current issues related to research in early intervention for children with ASD, implementation of evidence based models in the real world, and future directions.

\section{Intensity of services provided}

Unfortunately, despite the well documented impact of early intervention, on average children with ASD in the U.S. begin intervention at approximately 5 years of age (Christensen et al., 2016). In countries outside the United States, when available, intervention starts on average at the same time (Devescovi et al., 2016). Additionally, the majority of children with ASD in lowand middle-income countries have no access to evidence based interventions (Divan, 2017). Therefore, most children with ASD miss a developmental window shown to improve outcomes (Vivanti et al., 2014). With the increase in the number of children being diagnosed, developing interventions that are appropriate to these young children 


\section{Current Challenges in Early Intervention for Children with Autism Spectrum Disorder (ASD): A Narrative Review}

that can begin immediately after diagnosis and that can support the needs of parents at a very difficult time in their lives is a national and international research priority (National Academy of Sciences-National Research Council, 2001). Currently, research evidence indicates that high intensity, long term interventions are the most effective in supporting development and diminish ASD symptoms and associated disabilities (Dawson et al., 2010; Lovaas, 1987; McEachin, Smith, \& Lovaas, 1993).

Although several Insurances and State Programs cover intensive interventions (25 hrs. per week), it is nonetheless very difficult to families of newly diagnosed children with ASD to access appropriate services. Barriers involve a continuous increase in the number of diagnoses as well as scarcity of specialized providers. Moreover, due to the high intensity of intervention currently recommended, it is hard for many children to find providers available immediately after diagnosis.

Accessing ASD intervention as soon as possible in life is a critical public health issue. While high intensity interventions currently represent the gold standard of services, it is clear that they cannot be accessed by all children with ASD in early development, immediately after diagnosis. Perhaps research and clinical practice may try to serve more children as early as possible through low intensity services by providing parent education. Parent based intervention have been successful in teaching children a variety of skills including verbal and non-verbal communication (Rogers et al., 2012), social interaction (Mahoney \& Perales, 2003), and play (Stahmer, 1995). Moreover, parents who actively participate in their children's intervention often report reduced depression and stress and increased self-efficacy (Estes et al., 2014). Additionally, by learning intervention strategies through professional coaching, the parents may be able to implement therapy throughout the day (Rogers et al., 2012). Therefore, the limited time spent with the professional may multiply and lead to many productive hours of parent-child interaction during the day, outside of the therapist-child setting.

\section{From Efficacy to Effectiveness}

The body of evidence supporting the positive impact of early intervention for children with ASD mainly comes from University based efficacy studies involving high levels of staff training and supervision and high intensity services (Vivanti et al., 2014). Methodologically rigorous studies evaluating efficacy usually require randomization of participants into experimental and control conditions as well as blind evaluation of treatment outcomes (Bowen et al., 2009). Randomization is often impossible to conduct in community based intervention due to the policies regulating the delivery of services and ethical issues. Moreover, blind evaluations are not usually conducted in clinical practice outside randomized control trials (RCTs).

Efficacy studies employing randomized control trials (RCTs) test whether a given intervention is efficacious under controlled conditions (Smith et al., 2007). Studies of generalizability to community practice are limited due to the difficulties involved in selection of participants and clinicians. Community effectiveness studies test whether an intervention is effective when administered in community settings without rigorous research oversight (Smith et al., 2007). In effectiveness studies it is not possible, nor desired, to maintain the level of experimental control that is characteristic of randomized clinical trials such as randomization of participants and strict inclusion and exclusion criteria since the goal is to determine whether an intervention would be effective as practiced by 


\section{Current Challenges in Early Intervention for Children with Autism Spectrum Disorder (ASD): A Narrative Review}

community clinicians. Due to the complexity involved in conducting good quality effectiveness studies, very few ASD specialized early interventions have been evaluated through community studies (Kaale et al., 2012; Divan et al, 2015).

A priority in the ASD field is to evaluate whether specialized interventions proven efficacious in University RCTs are effective when implemented in communities around the world. Many communities lack the resources needed for the implementation of rigorous efficacy studies. An additional challenge for many non- English -speaking communities is that most evidence- based early intervention models for young children with ASD have been developed in Englishspeaking countries. Using an intervention developed in a different country with a different language and different cultural contexts implies the translation of the intervention manual, as well as adaptations of procedures to the specific culture of a given community.

Efficacious intervention need to be accessible and effective in the real world. Population with potential counfounds are usually excluded in intervention studies. As a result we know very little about intervention efficacy and effectiveness in children with low income and/or migrant status, or children living in developing countries.

While all evidence-based early intervention have been developed and tested in high-income countries, the majority of children live in low income countries (Divan, 2017). Most children living in low income countries lack access to high quality evidence based intervention. Barriers include lack of resources needed to provide high intensity interventions as well as scarcity of specialized professionals (Patel et al., 2013). Interestingly, Divan et al., (2015) have recently published a randomized trial evaluating the adaption of a well-supported evidence based intervention. A key aspect that allowed implementation in a low resourced region of India included the delivery of a parent-mediated intervention through non specialist providers. In many underserved areas of the world providing high intensity therapist led intervention may not be realistic, especially if we hope to deliver early intervention to the majority of children with ASD. On the other hand, all children deserve to receive high quality evidence based intervention adapted and evaluated in their communities. Hopefully, it will be possible to conduct more research in low-income countries to develop and evaluate interventions that could serve more children and lead to good outcomes.

\section{Inclusion/Exclusion criteria}

Despite the overall documented positive impact of early intervention for preschoolers with ASD (12 - 72 months), response to intervention program is variable. Outcomes for preschoolers who received early intervention range from loss of diagnosis to lack of improvement in the core ASD symptoms, from dramatic gains in language, cognitive, and adaptive skills to minimal treatment gains (Howlin, Magiati, and Charman, 2009).

There are at least two possible reasons for the variability in the outcome of early intervention studies. First, most studies do not describe the sample characteristics in detail. Even less is mentioned about the social and demographic factors that might influence the outcome. Second, is the clinical heterogeneity of autism (Vivanti et al., 2014). Despite the current custom of conceptualizing autism as a spectrum disorder following the publication of DSM5 , it is almost certainly the case that subtypes exist within the autistic spectrum (see Rutter 2014). In addition to the possible 


\section{Current Challenges in Early Intervention for Children with Autism Spectrum Disorder (ASD): A Narrative Review}

subtypes of autism, several medical and behavioral conditions are known to co-exist with it. It is estimated that $75 \%$ of individuals with ASD present associated medical conditions, genetic syndromes, or mental health disorders (Nazeer and Ghaziuddin, 2012). Due to the attempt to recruit homogeneous samples of individuals with "pure" ASD, children with associated conditions such as epilepsy, severe intellectual disabilities, or genetic abnormalities, are not included in many intervention studies (Dawson et al, 2010). Thus, by excluding children with ASD who have associated medical and behavioral disorders - who, in fact, constitute the majority of the general ASD population these stringent inclusion and exclusion criteria significantly reduce the generalizability of results and reduce their utility in the real world. On the other hand, in studies where all children with ASD are included and comorbid conditions are not considered as moderators in the statistical analyses, it is not possible to evaluate whether associated medical conditions have an impact on outcomes.

\section{Can ASD be cured by behavioral intervention?}

Early behavioral intervention started after the upsurge of ASD symptoms - the current clinical and research practice - has demonstrated positive impact on outcomes, but does not seem to be the answer in terms of curing ASD. Randomized controlled trials have demonstrated that children with ASD receiving high quality early intervention immediately after diagnosis make significant improvements (Rogers et al., 2012). However, the vast majority of the children treated continue to meet criteria for an ASD diagnosis (Dawson et al., 2010).
Autism symptoms start to be evident in most children between 6 and 12 months. Starting intervention at 6 months or soon after, when development still has not derailed from a typical trajectory may be an option to explore in terms of preventing or curing ASD. Only two comparison studies have attempted these route so far (Rogers et al., 2014; Green et al., 2015). Both studies showed improvement in developmental outcomes as well as a decrease in ASD symptoms in the treated infants in comparison to a control group that did not receive intervention.

\section{Conclusion}

Early intervention for children with ASD is considered unanimously needed and efficacious by the scientific community. Early intervention in ASD is a field that has seen high level of productivity since Lovaas seminal work in the 70s. Despite a big bulk of knowledge produced through rigorous Universities based trials, still a lot of work is needed to answer basic research questions as well as to develop or adapt interventions that could serve more children as soon as possible in life. Interventions that begin during the first year of life may perhaps lead to dramatically change the course of the disorder by preventing the unfolding of symptoms. The inclusion of children with associated medical condition, previously usually excluded from research, will allow to evaluate the impact of comorbidity on intervention outcomes. Finally, the international research community should develop and/or adapt interventions accessible in low resources countries where the majority of children live. In order to reach those areas both quality and feasibility of the interventions should be taken into consideration. 
Medical Research Archives. Volume 5, issue 7. July 2017.

\section{Current Challenges in Early Intervention for Children with Autism Spectrum Disorder (ASD): A Narrative Review}

\section{References}

American Psychiatric Association. (2013). Diagnostic and statistical manual of mental disorders: DSM-5. Washington, D.C: American Psychiatric Association.

Christensen DL, Baio J, Braun KV, et al. Prevalence and Characteristics of Autism Spectrum Disorder Among Children Aged 8 Years - Autism and Developmental Disabilities Monitoring Network, 11 Sites, United States, 2012. MMWR Surveill Summ 2016;65(No. SS-3)(No. SS-3):1-23.

Devescovi, R., Monasta, L., Mancini, A., Bin, M., Vellante, V., Carrozzi, M., \& Colombi, C. (2016). Early diagnosis and Early Start Denver Model intervention in autism spectrum disorders delivered in an Italian Public Health System service. Neuropsychiatric Disease and Treatment, 12, 1379-1384.

Divan, G. (2017), Editorial Perspective: 'From there to here': adapting child and adolescent mental health interventions for low-resource settings. J Child Psychol Psychiatr, 58: 325-327.

Dawson, G., Rogers, S., Munson, J., Smith, M., Winter, J., Greenson, J., . . Varley, J. (2010). Randomized, controlled trial of an intervention for toddlers with autism: the Early Start Denver Model. Pediatrics, 125(1), e17-e23.

Estes, A., Vismara, L., Mercado, C., Fitzpatrick, A., Elder, L., Greenson, J., . . . Young, G. (2014). The impact of parentdelivered intervention on parents of very young children with autism. Journal of autism and developmental disorders, 44(2), 353-365.

Howlin, P., Magiati, I., \& Charman, T. (2009). Systematic review of early intensive behavioral interventions for children with autism. American Journal on Intellectual and Developmental Disabilities, 114(1), 2341.

Koegel, R. L., Bimbela, A., \& Schreibman, L. (1996). Collateral effects of parent training on family interactions. Journal of autism and developmental disorders, 26(3), 347-359.

Koegel, L.K., Koegel, R.L., Ashbaugh, K., \& Bradshaw, J. (2014). The importance of early identification and intervention for children with or at risk for autism spectrum disorders. International Journal of SpeechLanguage Pathology, 16, 50-56.

Lovaas O. I. Behavioral treatment and normal educational and intellectual functioning in young autistic children. Journal of Consulting and Clinical Psychology. 1987;55:3-9.

Mahoney, G., \& Perales, F. (2003). Using relationship-focused intervention to enhance the social - emotional functioning of young children with autism spectrum disorders. Topics in Early Childhood Special Education, 23(2), 74-86.

McEachin, J. J., Smith, T., \& Lovaas, O. I. (1993). Long-term outcome for children with autism who received early intensive behavioral treatment. American Journal of Mental Retardation, 97, 359-359.

Patel, P., Kieling, C., Maulik, P.K., \& Divan, G. (2013). Improving access to care for children with mental disorders: A global perspective. Archives of Diseases in Childhood, 98, 323-327.

Rogers S. J. et al. (2012) Effects of a brief Early Start Denver Model (ESDM) based parent intervention on toddlers at risk for autism spectrum disorders: A randomized controlled trial. Journal of the American 


\section{Current Challenges in Early Intervention for Children with Autism Spectrum Disorder (ASD): A Narrative Review}

Academy of Child and Adolescent Psychiatry. 51(10), pp. 1052-1065.

Stahmer, A. C. (1995). Teaching symbolic play skills to children with autism using pivotal response training. Journal of autism and developmental disorders, 25(2), 123141.

Vivanti G. et al. (2014) Effectiveness and feasibility of the Early Start Denver Model implemented in a group-based community childcare setting. Journal of Autism and Developmental Disorders. December, 44(12), pp. 3140-3153.

Wetherby AM, Guthrie W, Woods J et al. Parent-implemented social intervention for toddlers with autism: an RCT. Pediatrics 2014;134:1-10. 